\title{
Balkanologie
}

Balkanologie Revue d'études pluridisciplinaires

Vol. $16 n^{\circ} 1 \mid 2021$

Au-delà de la "route des Balkans ": mondes sociaux des circulations

\section{Écrire une histoire des Tsiganes balkaniques}

How to Write a History of the Balkan Gypsies?

\section{Bernard Lory}

\section{(2) OpenEdition}

Journals

\section{Édition électronique}

URL : https://journals.openedition.org/balkanologie/3073

DOI : 10.4000/balkanologie.3073

ISSN : 1965-0582

Éditeur

Association française d'études sur les Balkans (Afebalk)

Référence électronique

Bernard Lory, «Écrire une histoire des Tsiganes balkaniques », Balkanologie [En ligne], Vol. 16 n 1। 2021, mis en ligne le 01 juin 2021, consulté le 03 octobre 2021. URL : http://journals.openedition.org/ balkanologie/3073 ; DOI : https://doi.org/10.4000/balkanologie.3073

Ce document a été généré automatiquement le 3 octobre 2021.

(c) Tous droits réservés 


\section{Écrire une histoire des Tsiganes balkaniques}

How to Write a History of the Balkan Gypsies?

Bernard Lory

1 Un grand peuple balkanique (trois ou quatre millions de personnes?) n'a pas encore fait l'objet d'une synthèse historique : les Tsiganes ou Rroms ${ }^{1}$. Ce constat m'a poussé à un vaste travail de collecte et de commentaire des sources historiques disponibles. Ce travail préalable, indispensable à toute étude historique, est en voie de finalisation ${ }^{2}$. Le moment est donc opportun d'envisager la phase suivante : une histoire des Tsiganes balkaniques est-elle possible, dans le cadre de nos connaissances actuelles? Quels sont les principaux obstacles auxquels est confronté l'historien quand il aborde une matière aussi délicate?

Des causes, tant endogènes qu'exogènes, se laissent identifier dans un premier temps. D'une part, les Tsiganes n'ont manifesté aucun intérêt pour la chose écrite avant le $\mathrm{xx}^{\mathrm{e}}$ siècle. Ils n'ont donc pas produit la matière première indispensable à un récit historique: des archives. Ils n'ont pas constitué non plus de corpus oral à vocation mémorielle, comparable par exemple aux chants épiques des Slaves du Sud. À l'intérieur d'un groupe familial, la transmission intergénérationnelle dépasse rarement deux ou trois générations; elle s'appuie peu sur le récit biographique ou familial ${ }^{3}$. L'historien est tenté d'en conclure que les choses du passé n'ont guère de valeur aux yeux des Tsiganes...

3 D'autre part, la pratique historienne dans les Balkans relève des élites et est étroitement associée au projet national. Il s'agit de construire un grand récit collectif qui s'articule généralement sur le schéma: origines obscures et disputées, gloire et puissance médiévale, chute et oppression sous les Ottomans, réveil patriotique et perspective d'une nouvelle efflorescence. Les historiens ont été particulièrement mis à contribution dans la phase de compétition entre les peuples balkaniques, dans l'élaboration d'un argumentaire "scientifique " justifiant leurs micro-impérialismes. Les Tsiganes sont tout bonnement absents de ces grands récits constitutifs, comme ils 
le sont des polémiques interbalkaniques, car ce ne sont pas des compétiteurs. On peut donc les ignorer.

On n'a donc pas écrit à leur place l'histoire que les Tsiganes ont négligé d'écrire euxmêmes. Peut-être cela vaut-il mieux... Les Pomaks n'ont pas trop à se féliciter de la manière dont leur histoire a été traitée par des historiens pleins d'arrière-pensées ; les Macédoniens mènent d'interminables polémiques avec des historiens des pays voisins prétendant « mieux connaître le passé » qu'eux. Être anhistorique, être invisible dans des narrations essentiellement nationalistes, n'est-ce pas une forme de protection ${ }^{4}$ ? N'est-ce pas une manière de traverser le village en ayant quelques chiens de moins qui aboient à vos trousses?

\section{Un manque}

Dans une perspective balkanologique, la question se pose différemment. Pour commencer, il est contrariant qu'un fragment du puzzle reste vacant. La balkanologie a une vocation englobante et ambitionne de dresser un tableau du Sud-Est européen aussi complet et détaillé que possible : toute lacune réclame à être comblée.

Mais les Tsiganes sont aussi la principale population balkanique transétatique ; ils sont incomparablement plus nombreux et plus répandus que les Aroumains ou les Juifs. Ils contribuent à leur manière à ce substrat transbalkanique commun que les chercheurs ont tant de mal à mettre à jour derrière les discours clamant la spécificité de chaque groupe national. L'évanescent balkanisme ne serait-il pas plus facile à cerner à partir du prisme tsigane?

7 De prime abord, on peut identifier au moins deux aspects de l'histoire des Balkans qui s'éclairent différemment si on adopte une " perspective tsigane ».

Depuis que leur présence est connue dans les Balkans, les Tsiganes occupent l'échelon le plus bas de l'échelle sociale. On peut même dire que c'est la fonction qui leur est assignée et qu'ils assument. Dans l'échelle des valeurs ottomanes, un Turc est supérieur à un Pomak, un Grec à un Bulgare, mais un riche Pomak aura plus de poids qu'un pauvre Turc, un riche Bulgare qu'un pauvre Grec ; un riche Grec peut même s'aventurer à mépriser un pauvre Turc. Dans cette hiérarchisation ethnique, confessionnelle et économique, le Tsigane occupera toujours la position inférieure : un Tsigane, même musulman, sera inférieur à un chrétien pauvre; un riche Tsigane n'est jamais coopté par l'échelon supérieur, ni musulman, ni chrétien ${ }^{5}$.

Rejetés par tous, jamais reconnus ni comme de "vrais » musulmans ni comme de « vrais » chrétiens, les Tsiganes fournissent une référence négative commune à toutes les composantes de la société ottomane. Même réduits à la misère, musulmans ou chrétiens peuvent se consoler en soupirant : «Encore heureux, grâce à Dieu, que nous ne soyons pas tsiganes!». Cette référence commune à une abjection ethnicisée, qui transcende le clivage confessionnel structurant la société ottomane, fournit une sorte de socle auquel tous peuvent se référer. La frustration de la composante chrétienne, systématiquement rappelée à son statut d'infériorité par le système des millets, trouve un exutoire à pouvoir mépriser les Tsiganes. Leur rejet, amusé ou dédaigneux, condescendant ou agacé, permet même d'établir une forme de connivence entre musulmans, chrétiens et juifs en faisant ressortir les mérites d'un komşuluk interconfessionnel, dont les Tsiganes ne respectent pas les règles de bienséance ${ }^{6}$. 
L'étude d'une société par ses marges (subaltern studies) permet de faire ressortir des mécanismes de régulation tellement évidents qu'ils ne sont jamais énoncés.

Dans le cadre des États-nations en voie de modernisation, les Tsiganes ont, nous semble-t-il, une autre fonction: celle de conservatoire d'usages en voie d'extinction. Les anthropologues soulignent volontiers le conservatisme des groupes tsiganes. Ceuxci maintiennent en vie des éléments de culture balkanique que la population dominante tend à abandonner. $\mathrm{Au} \mathrm{Xx}^{\mathrm{e}}$ siècle, ils sont les derniers à pratiquer le rituel pour la pluie de dodola/peperuda, à souhaiter le Nouvel An des survakars, à porter des şalvars pour les femmes, etc. Dans la post-modernité du xxI ${ }^{\mathrm{e}}$ siècle, ils maintiennent des attelages de chevaux quand tout roule à l'essence, font de la musique de mariage quand triomphe la "musique en boîte ", font de la récupération dans une société de gaspillage, etc. Dans la course en avant qui entraîne les peuples balkaniques à un rythme toujours plus rapide, ils opposent une force d'inertie et en viennent à emblématiser une vie d'autrefois, pour laquelle on peut nourrir une certaine nostalgie.

11 Ceci ne signifie pas que la société tsigane soit immobile, loin de là, mais elle marque un certain décalage par rapport aux évolutions de la société ambiante. Pour l'historien, il y a là matière à réflexion. L'histoire des Tsiganes que nous voudrions écrire n'est pas synchrone avec l'histoire déjà écrite des peuples balkaniques. Cerner sa temporalité propre est un véritable défi.

12 La tsiganologie existe enfin comme champ de recherche scientifique ${ }^{7}$. La spécificité des modalités d'insertion des Tsiganes dans le contexte balkanique mérite réflexion. Le discours tsiganologique s'est formé en Europe occidentale et s'est focalisé sur certains aspects de la vie tsigane, comme le nomadisme (roulottes, aires de stationnement), la musique (flamenco, jazz manouche), la vie religieuse (Saintes-Maries-de-la-Mer), questions de pureté/impureté (spécifiques aux groupes kalderaš et assimilés), etc. En cherchant à définir une universalité tsigane, on s'est attaché à des aspects pertinents pour l'Europe occidentale, mais qui le sont beaucoup moins pour l'Europe balkanique. Or le centre de gravité démographique des Tsiganes européens est dans le sud-est du continent...

13 Prenons le cas du nomadisme. Si les Tsiganes d'Europe centrale, relativement peu nombreux, adoptent ce mode de vie en s'éparpillant dans des régions densément peuplées, les Tsiganes balkaniques sont, pour leur part, essentiellement sédentaires ; ils occupent un quartier spécifique à la périphérie des villes et des villages, ce qui ne les empêche pas de faire des tournées saisonnières à partir de ce lieu d'habitation durant lesquelles ils vivent sous la tente. Saupoudrage et concentration sont deux stratégies de survie différentes, mises en œuvre dans des contextes qu'il faut analyser8.

14 Signalons aussi que, dans les Balkans, un nombre non négligeable de Tsiganes était engagé à temps plein dans l'activité agricole, dans les grands domaines fonciers à la fin de l'époque ottomane comme dans les coopératives agricoles de l'époque communiste. Mais ce qui interroge le plus l'historien, c'est le quasi-monopole sur le métier de forgeron/maréchal ferrant dont ils jouissent au xIX ${ }^{e}$ siècle. Comment un secteur aussi vital pour l'économie rurale a-t-il pu échapper aux populations dominantes ${ }^{9}$ ? Des rapports d'interdépendance étroits ont existé dans les Balkans, qui n'ont pas d'équivalents en Europe occidentale ${ }^{10}$.

15 La recherche tsiganologique a massivement réinvesti le champ balkanique après 1990 . Elle découvre une situation calamiteuse, la déstructuration générale de la société dans 
la «transition économique", se répercutant de façon implacable sur tous les marginaux. Le "présentisme » dans lequel vit notre époque fait table rase de toutes les expériences de cohabitation entre Rroms et non-Tsiganes (Gadjos ${ }^{11}$ ) de par le passé. L'absence d'approche historique tend à essentialiser le malheur tsigane, comme si le désastreux xxI ${ }^{\mathrm{e}}$ siècle était la règle et non l'exception.

\section{La langue comme source}

La "matière historique " tsigane constitue un défi particulier pour l'historien. Peuple sans archives, presque sans mémoire orale, ne laissant pas de vestiges archéologiques, il a pour principal patrimoine sa langue, le rromani, dont on sait depuis la fin du $\mathrm{XVIII}^{\mathrm{e}}$ siècle qu'elle vient d'Inde. C'est donc ce fil-là que la recherche scientifique a tiré pour remonter dans le passé d'un peuple mystérieux. Déterminer une région d'origine et une date de départ a été compliqué et contradictoire, car la culture historique est tardive en Inde même, où manquent cruellement les sources écrites avant l'arrivée de l'islam ${ }^{12}$. Diverses théories ont été proposées. Celle qui recueille le plus grand assentiment de nos jours propose la vallée du Gange comme région d'origine et les raids dévastateurs de Mahmud de Ghazni, au début du XI ${ }^{\mathrm{e}}$ siècle, comme moteur initial des migrations. Le saccage de la ville de Kannaoudj en 1018 devient le moment-zéro symbolique de l'expansion tsigane. Le cheminement des proto-Rroms à travers le Moyen et le Proche-Orient reste toutefois entouré des brumes les plus profondes. Pour étoffer cette trame chronologique extrêmement précaire, la linguistique fournit quelques éléments précieux. À une date précoce, les parlers des Loms (ou Poshas) d'Arménie divergent, de même que ceux des Doms (ou Nawars) du Proche-Orient. La migration européenne concerne exclusivement le rameau des Rroms, dont les parlers se caractérisent par une forte présence de lexique grec et une quasi-absence de lexique arabe.

17 La première attestation indiscutable d'une présence tsigane dans l'Empire byzantin remonte aux années $1280^{13}$. Des attestations fiables, mais très ponctuelles, se retrouvent en Crète en $1323^{14}$ et à Dubrovnik en $1362^{15}$. La date de 1417 est généralement admise comme celle de l'arrivée des Tsiganes en Europe centrale et occidentale ${ }^{16}$.

Dans une phase ultérieure, une coupure dialectale s'établit en Europe, séparant les parlers de Roumanie, d'Hongrie, de Croatie, d'Italie du Sud et de tout le Sud-Est européen de ceux du reste du continent. Cette ligne suit à peu près celle de l'extension maximale de l'Empire ottoman (théorie du Great Divide ${ }^{17}$ ). Mais la documentation historique dont on dispose a du mal à confirmer le constat fait par les linguistes. Les circulations tsiganes s'interrompirent-elles vraiment? Les frontières politico-militaires étaient-elles si hermétiques?

19 Vers le milieu du $\mathrm{XIX}^{\mathrm{e}}$ siècle, une vague migratoire fortement caractérisée dialectalement part des pays roumains et essaime à travers l'Europe et la planète entière (migration kalderaš). Là encore, et quoiqu'on soit à une époque très proche où les sources écrites sont a priori abondantes, la documentation historique s'ajuste mal aux données linguistiques. Les causes et les modalités de la migration kalderaš restent mal connues. On met souvent en avant l'abolition de l'esclavage des Tsiganes en Valachie et en Moldavie, mais c'est une explication en trompe-l'œil : la concomitance géographique et chronologique n'est pas, en elle-même, une explication. 
20 La langue n'est cependant pas l'unique fil conducteur que peuvent suivre les chercheurs. Il y a aussi la farouche volonté d'altérité des Tsiganes, laquelle se combine à la discrimination pratiquée par la société dominante, comme les deux faces d'un même phénomène. Placés au plus bas de l'échelle sociale, les Tsiganes, même ceux qui atteignent une certaine prospérité à l'époque ottomane, ne brisent pas les lois de l'endogamie. Le choix d'un conjoint non tsigane n'est pas souhaitable. Si cela se produit, la société ambiante y voit une lourde mésalliance (hypogamie) et considère son membre (une femme le plus souvent) comme perdu pour la communauté. L'ascension sociale par le biais du mariage (hypergamie), qui a été un fort facteur de brassage socioethnique dans les Balkans ${ }^{18}$, ne joue donc pas pour les Tsiganes ${ }^{19}$.

21 Cette ligne de fracture (altérité assumée/rejet) constitue un fil ténu que l'historien peut tirer, car il en trouve trace dans la documentation produite par la société dominante. C'est sur cette base que peut se construire, malgré tout, une histoire des Tsiganes dans les Balkans. Si les Tsiganes étaient seulement des pauvres, ils n'auraient pas laissé de traces dans la maigre documentation balkanique; dans la mesure où ce sont des "pauvres différents", ils figurent dans les sources, pourvu que l'historien sache où chercher.

\section{Sources écrites}

La documentation historique éclaire le fait tsigane de façon différente selon les époques et selon les producteurs d'archives. Les très rares sources médiévales émanent d'ecclésiastiques qui se posent la question : ce peuple étrange qui se livre à la divination (chiromancie, oniromancie) est-il assimilable à des magiciens ou des hérétiques, dangereux pour le salut des fidèles ? Le nom populaire d'Athinganoi qu'on leur a assigné dans l'Empire byzantin se réfère à une hérésie, attestée au $\mathrm{IX}^{\mathrm{e}}$ siècle, disparue à l'époque de leur arrivée, mais dont le nom circulait encore ${ }^{20}$.

Des pèlerins occidentaux, en route vers Jérusalem, sont frappés par l'altérité des Tsiganes en Crète dès 1323, surtout à l'escale de Modon dans les années 1470-1500, où une importante colonie, installée hors des murs de la ville, est fréquemment décrite ${ }^{21}$. Le cas spécifique de Corfou, où exista du Xve siècle jusqu'à la fin du XvIII siècle un "Fief des Tsiganes » (Feudum Acinganorum), mériterait une étude approfondie s'appuyant sur les riches archives de l'île.

Avec l'instauration du régime ottoman, l'information change de nature et devient un peu plus abondante. Des Tsiganes apparaissent dans les registres de timars, ziyamets et hass, plus rarement dans les registres de vakifs. Les mentions les plus anciennes datent de la première moitié $\mathrm{du} \mathrm{xv}^{\mathrm{e}}$ siècle. On dispose d'un document exceptionnel avec le registre des Tsiganes de Roumélie pour $1523^{22}$, qui fournit le nom de tous les chefs de cemaats et le nombre de mâles adultes et de veuves pour chaque cemaat. Si les Tsiganes sont expressément désignés comme tels dans les sources ottomanes, c'est qu'ils constituent une catégorie fiscale spécifique. Quoiqu'ils ne rapportent guère aux caisses de l'État, ils font l'objet d'un enregistrement séparé, ce qui n'a pas d'équivalent en Europe occidentale. La fiscalité spécifique aux Tsiganes est connue à partir du règne de Mehmed $\mathrm{II}^{23}$; on est particulièrement bien informé pour le $\mathrm{XVII}^{\mathrm{e}}$ siècle. Rédiger une histoire des Tsiganes de l'Empire ottoman à partir de ces sources fiscales est un projet parfaitement envisageable, qui n'attend que des chercheurs courageux. 

l'étude commence à enrichir nos connaissances. Les yoklama defteris (registres des contribuables absents) ${ }^{31}$ et les temettüat defteris, qui établissent les revenus des citadins, fournissent des éclairages très précis ${ }^{32}$. L'expérience-pilote de Vilayet du Danube a laissé une grosse documentation ${ }^{33}$. Enfin, pour les dernières décennies de l'Empire, les annuaires provinciaux (salnames) sont les seules sources indiquant une évaluation numérique de la population tsigane (lorsque les fonctionnaires étaient consciencieux !). Une réflexion tsiganologique ottomane s'esquisse à la fin $\mathrm{du}_{\mathrm{XIX}}{ }^{\mathrm{e}}$ siècle; elle est très tributaire de la recherche occidentale ${ }^{34}$.

Un autre fil historique que l'on peut tirer est celui de l'islamisation. Les registres nominaux permettent d'établir la proportion de chrétiens et de musulmans et les stratégies familiales de conversion, grâce aux «fils d'Abdallah» qui signalent des convertis de première génération.

l'administration ottomane. Il semblerait qu'au moment de la conquête, les gazis ottomans aient recouru à des auxiliaires tsiganes musulmans venus d'Anatolie. Pour les distinguer des Tsiganes chrétiens balkaniques, une entité administrative, le Sancak des Tsiganes, localisée en Thrace orientale, leur a été dévolue ${ }^{24}$. Les conversions à l'islam se multipliant, le pouvoir ottoman s'est efforcé d'obtenir une ségrégation spatiale et matrimoniale entre "croyants » et "infidèles ", dont les Tsiganes ne tiennent aucun compte. Pour sortir de cette contradiction, les agents du fisc en viennent à prendre une mesure contraire à la şeriat en faisant payer le haraç aux Tsiganes musulmans; ils l'établissent à un taux légèrement inférieur à celui des chrétiens. surtout pour des affaires de maintien de l'ordre. L'exploitation de ce type de sources détermine une approche très foucaldienne de "surveiller et punir $»^{25}$; elle entre en résonnance avec la recherche tsiganologique occidentale qui s'appuie beaucoup sur des sources de ce genre. Cette approche, à tendance victimaire, est souvent reprise par les ONG qui défendent les droits des Rroms. On en retire l'image d'un peuple obstinément et férocement persécuté. Or les injonctions des mühimme defteris sont répétitives et stéréotypées, pour ne pas dire incantatoires, et il ne faut pas nécessairement les prendre au pied de la lettre. celui d'une province, mais surtout elle montre comment, à l'échelon local, toutes sortes d'interactions fines se négocient dans la vie quotidienne. On y trouve des actes de vente, des actes de mariage, des questions de succession, des règlements corporatifs ou municipaux, des litiges et des affaires criminelles. La recherche tsiganologique a commencé à défricher les sicils de Sofia ${ }^{26}$, Bitola ${ }^{27}$, Sarajevo ${ }^{28}$, Thessalonique ${ }^{29}$, etc., mais on est encore loin d'un dépouillement exhaustif. Des fragments de sicils pour Jajce et Tešanj montrent que, localement, la « question tsigane » peut être gérée de façon très variable $^{30}$. C'est certainement le gisement d'information le plus prometteur pour les Balkans entre le milieu du XVI et la fin du XVIII ${ }^{e}$ siècle.

L'Empire ottoman des Tanzimats produit toute une documentation administrative dont On peut compléter l'information ottomane avec les récits des voyageurs occidentaux. On y trouve, certes, la plupart des stéréotypes attendus, mais parfois aussi une rencontre ou une anecdote circonstanciée qui fournit un éclairage intéressant. Les sources émanant des milieux chrétiens balkaniques restent très maigres jusqu'à la fin 
du XviII ${ }^{\mathrm{e}}$ siècle ; elles émanent des milieux ecclésiastiques orthodoxes, qui ne semblent pas « voir » les Tsiganes ${ }^{35} \ldots$

31 À partir du XIX ${ }^{e}$ siècle, le tableau change de façon significative. Les sources balkaniques chrétiennes deviennent plus abondantes. Ce sont, d'une part, les premiers documents émanant des jeunes États balkaniques en voie d'émancipation. Le cas de la Serbie a été étudié en détail par Tihomir Djordjevićs ${ }^{36}$. Ce sont, ensuite, les sources autobiographiques, dont certaines, très connues, font partie de l'histoire littéraire (prota Matija Nenadović, Pera Todorović, Zaharij Stojanov, Athanasios SuliotisNikolaïdis) ; mais c'est souvent chez des auteurs secondaires qu'émergent les anecdotes les plus révélatrices (Nićefor Ninković, pop Minčo Kănčev, etc.). Dans une entreprise aussi importante que de raconter sa propre vie, il n'est pas «normal» de faire figurer des marginaux tels que les Tsiganes, qui devraient « normalement » rester invisibles. Si la narration l'exige, ce n'est pas seulement l'occasion de raconter une «bonne histoire " pour égayer le lecteur. C'est que la confrontation avec les Tsiganes est l'indice d'une contradiction sociale subtile. Les sources balkaniques éclairent ces mécanismes fins avec beaucoup de subtilité.

Deux études scientifiques rédigées par des auteurs balkaniques ont enrichi la recherche tsiganologique qui, jusque-là, s'était focalisée sur l'Europe centrale et occidentale. Le médecin stambouliote Alexandros Paspatis (1814-1891) publie Études sur les Tchinghianés en 1870, première étude exhaustive d'un parler rromani, qui tranche sur la pratique précédente consistant à rassembler des bribes de dialectes divers, recueillies dans des tavernes ou en prison. Paspatis est le premier à adopter une démarche active : il va dans les périphéries urbaines misérables et recueille, sous la tente, les propos de ses informateurs; il déjoue habilement les obstacles d'une enquête linguistique menée dans un milieu très polyglotte.

33 Alors que l'ethnologie balkanique des $\mathrm{XIX}^{e}$ et $\mathrm{XX}^{e}$ siècles est essentiellement une science du «nous autres» national, Tihomir Djordjević (1868-1944) consacre sa thèse à l'« Autre proche », celui que l'on côtoie tous les jours sans vraiment le regarder ${ }^{37}$. Il sait combiner enquête de terrain et recherche en archives, ce qui donne à son travail une profondeur chronologique, qui manque cruellement à tant d'études ethnographiques.

Avant la Première Guerre mondiale, quelques articles abordent la présence tsigane en Croatie et en Bosnie-Herzégovine. En revanche, les Tsiganes de Grèce, Bulgarie, Macédoine et Albanie restent encore largement inétudiés. Il faut signaler le rôle du Journal of the Gypsy Lore Society, qui répertorie bon nombre de sources. C'est le principal creuset où s'élabore une réflexion tsiganologique scientifique, même si l'approche historique est y faiblement représentée et si les Balkans n'y occupent qu'une place modeste.

Avec la disparition des empires et l'établissement d'États nationaux, le cadre sociopolitique dans lequel évoluent les Tsiganes balkaniques se trouve profondément modifié. Les exigences de la société dominante se font plus contraignantes. Outre la fiscalité et l'affiliation religieuse (principales exigences de l'époque ottomane) apparaissent la scolarisation, le service militaire et la participation aux guerres, les règlements d'urbanisme, les papiers d'identité, les obstacles aux frontières, etc. La croissance démographique rapide de la paysannerie balkanique réduit drastiquement les "communs», sur les ressources gratuites desquels s'appuyait une partie de l'économie tsigane (pâturages, osier, bois blanc). Les anciens métiers sont peu à peu étranglés par la concurrence industrielle. Il faut sans cesse trouver des niches nouvelles 
permettant à la fois de survivre économiquement et de préserver sa marge d'autonomie identitaire. S'il est relativement facile de suivre ces processus du point de vue de l'État producteur de textes normatifs, comprendre les stratégies d'adaptation tsiganes est plus complexe. Les travaux des ethnologues et anthropologues se révèlent alors très précieux.

Les contraintes exercées par l'État se renforcent sous le communisme. Elles vont jusqu'au changement d'état civil obligatoire pour les Tsiganes musulmans de Bulgarie en 1961-1962 ${ }^{38}$. La scolarisation peut être perçue comme une contrainte ; c'est aussi une échappatoire émancipatrice pour ceux qui savent profiter des mesures de discrimination positive. Lorsque le mouvement tsigane international commence à se constituer dans les années 1970, ce sont les représentants yougoslaves, roumains et bulgares qui en assurent le leadership. L'encadrement médical que garantissent les régimes communistes réduit sensiblement la mortalité infantile. Le décalage chronologique de la transition démographique des Tsiganes par rapport à la société dominante est à l'origine d'un phantasme nouveau, celui de la « tsiganisation » du pays.

Seule la Yougoslavie du titisme finissant a produit une recherche tsiganologique plus approfondie, essentiellement menée par des sociologues ${ }^{39}$. Après la chute des régimes communistes, les recherches se sont multipliées, en bonne partie grâce à l'impulsion financière des programmes internationaux ${ }^{40}$. L'anthropologie sociale est venue renforcer la sociologie. Son approche se situe cependant trop souvent «ici et maintenant ». Les Tsiganes interrogés répondent classiquement à l'enquêteur : « On a toujours fait comme ça ». Si l'enquête anthropologique ne va pas plus loin, l'historien n'y trouve pas son compte ${ }^{41}$. La profondeur historique des évolutions contemporaines est trop rarement questionnée $e^{42}:$ il y a là un véritable problème d'interdisciplinarité sur lequel il conviendrait de se pencher.

D'objet d'étude, les Tsiganes, qui depuis les années 1970 revendiquent l'ethnonyme de Rroms, sont devenus des sujets à part entière. L'intelligentsia rrome qui avait émergé sous les régimes communistes a été accaparée après 1989 par l'activisme des ONG, lesquelles ont proliféré et prolifèrent toujours. Elle a du mal à s'autonomiser sur le plan scientifique et reste très tributaire de la recherche pratiquée par les Gadjos. L'urgence sociale liée à la dégradation dramatique des conditions de vie quotidienne accaparent les compétences et les énergies. La recherche scientifique contemporaine n'accorde qu'une place restreinte à la dimension historique; le génocide commis pendant la Deuxième Guerre mondiale a focalisé beaucoup d'efforts ${ }^{43}$. La Roumanie a produit les études les plus intéressantes ${ }^{44}$.

La recherche historique sur les Tsiganes balkaniques a fait émerger quelques débats importants. L'arrivée des Tsiganes a-t-elle précédé la conquête ottomane ou en est-elle un sous-produit? Les Tsiganes ont-ils joui d'un statut privilégié et d'une bienveillance particulière dans l'Empire ottoman? Quelle est leur position dans le système des millets? Quelle est leur attitude dans la lutte pour l'indépendance des peuples balkaniques ${ }^{45}$ ? Comment les jeunes États balkaniques traitent-ils leurs minorités tsiganes? Ces questions ont été posées de façon un peu prématurée, avant qu'un socle documentaire solide n'ait été rassemblét ${ }^{46}$. Elles ne concernent pas, à proprement 
parler, les problématiques internes aux communautés tsiganes, mais se focalisent sur les rapports entre Tsiganes et Gadjos. Cet obstacle est bien difficile à lever, dans les conditions actuelles de connaissance du dossier.

Dans une approche d'histoire interne aux Rroms, les circulations transdanubiennes restent très mal connues : dans quelle mesure la pratique de l'esclavage en Valachie et Moldavie se répercute-t-elle sur les territoires voisins? La question majeure des migrations kalderaš au XIX ${ }^{e}$ siècle demande à être éclairée. L'apparition de frontières nouvelles modifie-t-elle les comportements migratoires (saisonniers, à moyen terme) ? Comment la hiérarchisation des groupes tsiganes les uns par rapport aux autres s'établit-elle, comment évolue-t-elle?

41 La documentation historique sur les Tsiganes balkaniques souffre d'être très émiettée. Elle se présente rarement sous forme de séries documentaires permettant de faire ressortir des évolutions. D'une région à l'autre, les stratégies d'ajustement à la société ambiante varient souvent: les informations recueillies en Bosnie ne valent pas nécessairement pour la Bulgarie... Il faut donc élargir la base documentaire et collecter toutes les indications sporadiques qui se peuvent rencontrer, même les plus ténues. Les Tsiganes constituent rarement le propos principal d'un document; ils apparaissent dans les marges du récit, dans un contexte souvent anecdotique. Beaucoup de stéréotypes sont inlassablement répétés de source en source, et l'historien doit faire preuve de subtilité pour en extraire un peu de neuf.

42 Notre conviction est, qu'en resituant les Tsiganes dans une histoire longue, sur plusieurs siècles, on fera ressortir leurs dynamiques internes et leur capacité d'adaptation aux changements de leur environnement. Loin de l'image stéréotypée d'un peuple anhistorique, isolé dans une bulle inaltérable, ils ont toujours entretenu des relations avec les sociétés environnantes: c'est cette dialectique que l'historien peut décrire et analyser. Ces évolutions sont peu visibles, car lentes, et n'épousent pas la périodisation admise dans les Balkans. Le regard des Gadjos est toujours prêt à noter les traits qu'il croit déjà connaître, mais il rechigne à toute révision des idées préconçues.

$43 \mathrm{Si}$, trente ans après la chute des régimes communistes et malgré l'engagement et le dévouement des nombreuses ONG, la "question tsigane " suscite trop souvent un sentiment de découragement et de fatalité, une meilleure connaissance des mécanismes du passé pourrait apporter quelque réconfort. L'histoire, quoi que tiennent les nationalistes, peut avoir une fonction thérapeutique pour les sociétés balkaniques...

\section{NOTES}

1. Depuis les années 1970, l'endonyme rrom est promu dans une démarche militante. L'historien peut utiliser le terme tsigane, qu'il trouve dans les sources, sans lui conférer de valeur péjorative. 2. À paraître aux éditions Les Belles Lettres. Titre provisoire : Matériaux pour une histoire des Tsiganes des Balkans 1280-1914. Notre propos exclut la Roumanie, où l'histoire des Tsiganes est très spécifique. 
3. Le sous-ensemble des Lovaris fait exception sur ce plan (MARUŠIAKOVA Elena, POPOV Veselin, Ciganite v Bălgarija [Les Tsiganes en Bulgarie], Sofia, Klub '90, 1993, p. 140).

4. scotт James, Zomia, ou l'art de ne pas être gouverné, Paris, Seuil, 2013, p. 309-313.

5. Une lecture attentive des sources montre qu'un courant « assimilationniste » a toujours existé parmi les Tsiganes balkaniques, mais ses efforts sont généralement réduits à néant par l'assignation à une image archétypale du Tsigane nomade, incontrôlé, sale, menteur, etc.

6. Cela s'illustre dans de nombreux proverbes communs aux langues balkaniques, $c f$. ІкоNOMOV Nikolaj, Balkanska narodna mădrost. Usporedici na bălgarski, srăbski, turski, rumănski, grăcki i albanski poslovici i pogovorki [Sagesse populaire balkanique. Parallélismes entre des proverbes et des dictons bulgares, serbes, turcs, roumains, grecs et albanais], Sofia, BAN,1968.

7. «Parmi l'abondante production de livres ou d'articles concernant les Tsiganes, il en est relativement peu qui fassent une place à leur histoire » (VAUX DE FOLETIER François de, Mille ans d'histoire des Tsiganes, Paris, Fayard, 1970, p. 8). La meilleure synthèse actuellement disponible est celle d'Angus FRASER, The Gypsies, Oxford, Wiley Blackwell,1995.

8. Les immenses bidonvilles qui se sont développés depuis la chute du communisme sont un cas extrême ; ils s'inscrivent néanmoins dans un processus long, que l'historien doit s'appliquer à reconstituer.

9. La littérature ethnographique observe le phénomène, mais n'apporte pas de réponses, $c f$. p. ex. KANEV Konstantin, Minaloto na selo Momčilovci, Smoljansko [Le passé du village de Momtchilovtsi, près de Smolyan], Sofia, Izdatelstvo na Otečestvenija front, 1975, p. 560-561 ; PRIMOVSKI Atanas, «Kovačite Agupti v gr. Madan» [Les forgerons Agouptes dans la ville de Madan], Izvestija na etnografskija institut i muzej [Nouvelles de l'institut ethnographique et du musée], II, Sofia, 1955, p. 217-265, etc.

10. Aucune recherche n'a été faite sur la fonction de kehaja ou seoski ciganin, homme à tout faire et crieur public, embauché par la communauté villageoise, $c f$. MARINOv Dimităr, Živa starina, t. IV, Običajno pravo [Antiquité vivante, t. IV, Droit coutumier], Rousse, 1894, p. 374-377.

11. Le pluriel, en rromani, est Gadje. Les militants d'organisations non gouvernementales (ONG) y recourent volontiers, mais ce n'est pas l'usage scientifique: on ne parle pas de Pomaci ni de Türkler.

12. MIKLOSICH Franz, Über die Mundarten und die Wanderungen der Zigeuner Europas (Denkschriften der kais. Akademie der Wissenschaften, Philosophisch-historische Klasse), I-XIII, Wien, 1872-1881.

13. La lettre du patriarche Grégoire II au grand logothète Théodore Muzalon n'est connue que depuis les années 1990, cf. Rосноw Ilse, мAтschкE Klaus-Peter, "Neues zu den Zigeunern im byzantinischen Reich um die Wende vom 13. zum 14. Jahrhundert », Jahrbuch der österreichischen Byzantinistik, XLI, Vienne, 1991, p. 241-254.

14. Golubovich Girolamo (Ofm), Biblioteca bio-bibliografica della Terra santa e dell'Oriente francescano, t. III, (1300-1332), Firenze, Quaracchi presso, Collegio di S. Bonaventura, 1919, p. 237-282.

15. TADIĆ Jorjo, Pisma $i$ uputstva dubrovačke republike. (Izvori za istoriju južnih Slovena) [Correspondance officielle et instructions de la République de Dubrovnik (Sources pour l'histoire des Slaves du Sud)], t. II, vol. 1, Belgrade, Srpska kraljevska akademija 1935, p. 101.

16. C'est la date que fournit la Cosmographie de Sebastian Münster (1550). Quelques indices légèrement antérieurs ont été repérés.

17. MATRAS Yaron, I Met Lucky People. The Story of the Romani Gypsies, Londres, Penguin Books, 2014.

18. LORY Bernard, «Immigration et intégration sociale à Plovdiv au XIX siècle », Revue des mondes musulmans et de la Méditerranée, $\mathrm{n}^{\circ}$ 66, 1993/94, p. 95-103.

19. L'exclusion sociale n'empêche pas un fort brassage génétique: la prostitution est une ressource que ne s'interdisent pas les Tsiganes balkaniques, à la différence de ceux d'Europe occidentale. Un kanunname de 1530 réglemente la prostitution des Tsiganes des grandes villes sur 
la Via Militaris (Orta Kol). Les voyageurs occidentaux des XVI-XVIII ${ }^{\mathrm{e}}$ siècles y recourent explicitement.

20. soulis George, "The Gypsies in the Byzantine Empire and the Balkans in the Late Middle Ages ", Dumbarton Oaks Papers, XV, 1961, p. 143-165.

21. WINSTEDT Eric Otto, "The Gypsies of Modon and the "Wine of Romeney" ", Journal of the Gipsy Lore Society, $2^{\mathrm{d}}$ s, III, 1909-1910, p. 57-69.

22. STOJANOvSKi Aleksandar, "Romite na balkanskiot poluostrov » [Les Rroms de la péninsule balkanique], Prilozi na Makedonskata Akademija na Naukite i Umetnostite, VII, 1, Skopje, 1976, p. 33-76.

23. BELDICEANU Nicoară, Les actes des premiers sultans conservés dans les manuscrits de la Bibliothèque Nationale de Paris. t. I. Actes de Mehmed II et Bayezid II, Paris-La Haye, Mouton and co, 1960, p. 102-104.

24. Son rôle et son fonctionnement restent mal connus. Le Sancak des Tsiganes semble avoir disparu à la fin du XVII ${ }^{\mathrm{e}}$ siècle.

25. CELIK Faika, « Exploring Marginality in the Ottoman Empire : Gypsies or People of Malice (Ehl-i Fesad) as Viewed by the Ottomans ", European University Institute Working Papers, Robert Schuman Center for Advanced Studies n²004/39, Fiesole, 2004, p. 161-182.

26. GĂLĂBOV Gălăb, DDUDA Herbert, Die Protokollbücher des Kadiamtes Sofia, München, R. Oldenbourg, 1960.

27. Turski dokumenti za istorijata na makedonskiot narod [Documents turcs sur l'histoire de la nation macédonienne] t. I-IV, Skopje, Komisija za publikuvanje na arhivska graga, 1963-1972.

28. Mujıć Muhamed, «Položaj Cigana u jugoslovenskim zemljama pod osmanskom vlašću » [Le statut des Tsiganes dans les pays yougoslaves sous l'empire ottoman], Prilozi orientalne filologije, III-IV, Sarajevo, 1952-1953, p. 137-193.

29. GINIO Eyal, « Neither Muslims nor Zimmis: the Gypsies (Roma) in the Ottoman State », Romani Studies, $5^{\text {th }}$ s, XIV, 2, 2004, p. 117-144.

30. TRUHELKA Ćiro, «Pabirci jednog jajačkog sidžila » [Fragments des minutes d'un greffier de Jajce], GZMBH, XXX, 1918, p. 157-174; ČAR-DRNDA Hatidža, Sidžil Tešanjskog kadiluka (1740-1752) [Le greffier du Tribunal de Tešan (1740-1752)], Sarajevo, Orijentalni Institut u Sarajevu, 2005.

31. G'ORG'IEV Dragi, Ohrid i Ohridsko niz osmanliskata dokumentacija (XIX-XX vek) [Ohrid et sa région à travers la documentation ottoman ( $\left.\mathrm{xIX}^{\mathrm{e}}-\mathrm{Xx} \mathrm{e}\right]$, Ohrid, 2009.

32. DINGEÇ Emine, «Ottoman Gypsies and the Head-Tax Collection: a Case Study of the Gypsies Living in Silistre in 1843», Türk-Islâm Medeniyeti Akademik Araştırmalar Dergisi, Konya, 2007, nº 4, p. 211-229; Osmanlı arşiv belgelerinde Kosova vilayeti [La province du Kosovo dans les documents d'archives ottomans], Osmanlı Arşivi Daire Başkanlığı, Istanbul, 2007.

33. DRAGANOvA Slavka, "Statistical Data about the Property and Taxes Paid by the Gypsy (Roma) Population of the Danube Province ", Bulgarian historical review, XXXVII, 3-4, 2009, p. 165-176.

34. ULUSOY Ömer, Les êtres en marge : les Tsiganes de l'Empire Ottoman, Istanbul, Isis Press, 2013 ; ÇELIK Faika, "Civilizing Mission" in the Late Ottoman Discourse: the Case of Gypsies ", Oriente moderno, XCIII, 2, 2013, p. 577-597.

35. LORY Bernard, «Les Tsiganes vont-ils au ciel ? », article à paraître dans les Cahiers Pierre Belon.

36. DJORDJEVIĆ Tihomir, "Ciganski haračluk u Srbiji », Naš narodni život ["L'imposition tsigane en Serbie », La vie de notre peuple], t. II, Beograd, Prosveta, 1984 [1928], p. 319-344.

37. GJORGJEVIĆ [sic] Tihomir, Die Zigeuner in Serbien, t. I-II, Budapest, Ethnologische Forschungen, 1903-1906.

38. STOYANOvA Plamena, Ciganite v godinite na socializma. Politikata na bălgarskata dăržava kăm ciganskoto malcinstvo (1944-1989) [Les Tsiganes pendant les années du socialisme. La politique de l'État bulgare à l'égard de la minorité tsigane (1944-1989)], Sofia, Paradigma, 2017, p. 75-97. 
39. Il faut signaler les apports historiques majeurs de Djurdjica PETROVIć : « Društveni položaj Cigana u nekim jugoslovenskim zemljama u XV i XVI veku» [Le statut social des Tsiganes dans certains des pays yougoslaves], Jugoslovenski istorijski časopis, 1-2, 1976, p. 45-66 ; ZIRoJEvić Olga, «Cigani u Srbiji od dolaska Turaka do kraja Xvi veka » [Les Tziganes en Serbie à partir de l'arrivée des Turcs jusqu'à la fin du XVI ${ }^{\mathrm{e}}$ siècle], Jugoslovenski istorijski časopis, 1-2, 1976, p. 67-77 ; eadem, « Romi na području današnje Jugoslavije u vreme turske vladavine » [Les Roms sur le territoire de l'actuelle Yougoslavie à l'époque du gouvernement turc], Glasnik etnografskog muzeja, XLV, 1981, p. 225-245.

40. CROWE M. David, A history of the Gypsies in Eastern Europe and Russia, New York, St. Martin's Press, 1994.

41. Des études ethnologiques réalisées il y a cent ans devraient pouvoir devenir des sources pour l'historien. Dans la pratique, cela s'avère difficile, car elles n'ont pas toujours été "outillées » pour resservir. Cette question de méthodologie n'a, à ma connaissance, pas été débattue.

42. Signalons la belle étude d'une anthropologue qui ne dédaigne pas la recherche en archive, Stefania PONTRANDOLFO, La dissolution identitaire d'une communauté rom. Ethnographie d'une disparition, Paris, L'Harmattan, 2013. Il ne s'agit malheureusement pas d'un terrain balkanique...

43. LENGEL-KRIZMAN Narcisa, «Prilog proučavanju terora u tzv. NDH : sudbina Roma 1941-1945 » [Contribution à l'étude de la terreur dans le soi-disant État indépendant de Croatie : le sort des Roms 1941-1945], Časopis za suvremennu povijest, 1, 1986, p. 29-42; ACKović Dragoljub, Stradanje Roma u Jasenovcu [La persécution des Roms à Jasenovac], Belgrade, ABC glas, 1994; AUZIAS Claire, Samudaripen, le génocide des Tsiganes, Paris, L’Esprit frappeur, 1999.

44. En particulier les travaux de Viorel ACHIM et Petre PETÇUȚ.

45. STOJANČEvić Vladimir, «Politički i pravni položaj Cigana (Roma) u Srbiji Prvog i Drugog Ustanka » [La situation politique et juridique des Tsiganes (Roms) en Serbie durant la première et la seconde insurrection], dans Razvitak Roma u Jugoslaviju. Problemi i tendencije [Le développement des Roms en Yougoslavie. Problèmes et tendances], Belgrade, Srpska akademija nauka i umetnosti, 1992, p. 25-30.

46. vuKANOvić Tatomir, Romi (Cigani) u Jugoslaviji, [Les Roms (Tsiganes) en Yougoslavie], Vranje, Štamparija nova Jugoslavija, 1983; MARUŠIAKOVA Elena, POPOV Veselin, Ciganite $v$ Osmanskata imperija [Les Tsiganes dans l'empire ottoman], Sofia, Litavra, 2000 ; BOŠKOVSKI Milan, GORGIEV Dragi, PETROVSKI Trajko, Istorija na Romite vo Makedonija i na Balkanot [Histoire des Roms en Macédoine et dans les Balkans], Skopje, Združenie na ljubiteli na romska folklorna umetnost « Romano Ilo », 2014 ; COURTHIADE Marcel, Petite histoire du peuple rrom, première diaspora historique de l'Inde, Lormont, Le Bord de l'eau éditeur, 2019.

\section{RÉSUMÉS}

Un grand peuple des Balkans ne dispose pas encore d'une histoire scientifique satisfaisante, les Tsiganes/Rroms. Cet article s'efforce de comprendre les raisons de cette lacune. Il répertorie les principaux gisements d'archives pouvant alimenter une documentation très déficiente. Il souligne la difficulté qu'a l'historien à travailler avec des études produites essentiellement par des linguistes et des anthropologues. 
An important people in the Balkans still has no written history of its own, the Gypsies/Rroms. This article tries to understand the reasons for this lacuna. It lists the main archival sources that can supplement this deficient field. It underlines the difficulties that a historian has in working with studies produced primarily by linguists and anthropologists.

INDEX

Mots-clés : Tsiganes, Rroms, tsiganologie, archives ottomanes, interdisciplinarité

Keywords : Gypsies, Roma, Romani studies, Ottoman archives, interdisciplinarity

\section{AUTEUR}

BERNARD LORY

INALCO, Paris 